\title{
Sulfolobus tokodaii RadA paralog, stRadC2, is involved in DNA recombination via interaction with $\mathrm{RadA}$ and $\mathrm{Hjc}$
}

\author{
WANG Lei ${ }^{1 \dagger}$, SHENG DuoHong ${ }^{1 \dagger}$, HAN WenYuan ${ }^{1}$, HUANG Bin ${ }^{1}$, ZHU ShanShan ${ }^{1}$, \\ NI JinFeng ${ }^{1}$, LI Jia ${ }^{2} \&$ SHEN YuLong ${ }^{1 *}$ \\ ${ }^{1}$ State Key Laboratory of Microbial Technology, Shandong University, Jinan 250100, China; \\ ${ }^{2}$ College of Pharmacy, Shandong University of Chinese Medicine, Jinan 250014, China
}

Received December 7, 2011; accepted February 16, 2012; published online March 22, 2012

\begin{abstract}
Rad51/RadA paralogs found in eukaryotes and euryarchaea play important roles during recombination and repair, and mutations in one of the human Rad51 paralogs, Rad51C, are associated with breast and ovarian cancers. The hyperthermophilic crenarchaeon Sulfolobus tokodaii encodes four putative RadA paralogs and studies on these proteins may assist in understanding the functions of human Rad51 paralogs. Here, we report the biochemical characterization of stRadC2, a S. tokodaii RadA paralog. Pull-down assays revealed that the protein was able to interact with the recombinase, RadA, and the Holliday junction endonuclease, Hjc. stRadC2 inhibited the strand exchange activity of RadA and facilitated Hjc-mediated Holliday junction DNA cleavage in vitro. RT-PCR analysis revealed that stRadC2 transcription was immediately reduced after UV irradiation, but was restored to normal levels at the late stages of DNA repair. Our results suggest that stRadC2 may act as an anti-recombination factor in DNA recombinational repair in S. tokodaii.
\end{abstract}

archaea, Sulfolobus, recombination, RadA, stRadC, Hjc

Citation: Wang L, Sheng D H, Han W Y, et al. Sulfolobus tokodaii RadA paralog, stRadC2, is involved in DNA recombination via interaction with RadA and Hjc. Sci China Life Sci, 2012, 55: 261-267, doi: 10.1007/s11427-012-4292-0

Rad51 paralogs are ubiquitous proteins in eukarya. In Saccharomyces cerevisiae, Rad55 and Rad57 proteins have been identified as Rad51 paralogs because of their high sequence homology to Rad51, and they form a heterodimer that facilitates formation of the functional Rad51 complex on RPA (replication protein A, RPA)-coated single-stranded (ss) DNA [1,2]. Genetic analysis suggested that Rad55 and Rad57 might play more important roles in the recombination repair (HR) of stalled replication forks than in DSB repair [3]. In contrast to yeast, five Rad51 paralogs (Rad51B, Rad51C, Rad51D, Xrcc2, and Xrcc3) have been identified in mammals. These proteins share $20 \%-30 \%$ sequence identity with Rad51 and with each other, and are

$\dagger$ Contributed equally to this work

*Corresponding author (email: yulgshen@sdu.edu.cn) thought to mediate DNA strand exchange events during HR [4-6]. The knockout mutants of each of the five Rad51 paralogs were viable in chicken B-lymphocyte DT40 cell lines, but showed spontaneous chromosomal aberrations, hypersensitivity to DNA-damaging agents, and low levels of HR [7].

It has been reported that archaeal genomes encode recombination repair proteins that are generally more similar to eukaryotic equivalents than to bacterial ones [8]. Accordingly, archaea possess recombinase RadA paralogs [9]. In euryarchaea, Pyrococcus furious has one RadA paralog, named RadB. The protein has a strong binding affinity for DNA and a very weak ATPase activity that is not stimulated by DNA [10]. In addition, RadB can interact with RadA directly and repress the recombination activity of RadA [11]. In Haloferax volcanii, deletion of $\mathrm{radB}$ results in growth 
retardation, increased sensitivity to UV light and recombination defects, suggesting a role of $\mathrm{RadB}$ as a recombination mediator [9]. The hyperthermophilic crenarchaea, another branch of archaea, has several RadA homologs, such as SSO2452, SSO1861 and SSO0777 in Sulfolobus solfataricus, and ST0579, ST0838, ST1830 and ST2522 in S. tokodaii [12-14]. These homologs were suggested to be RadA paralogs, because they have homology to RadA and some of them are involved in DNA repair, although their exact relationship with RadA has not been fully determined $[12,13]$. For convenience, in this study we will refer to these homologs as RadA paralogs. Interestingly, sequence alignment showed that some of these proteins, for instance, SSO2452 and SSO1861 in S. solfataricus, and ST0579 and ST1830 in S. tokodaii were more similar to the KaiC-like proteins than to any known $\operatorname{RadA} / \operatorname{Rad} 51$ paralog found throughout archaea and eukarya. Therefore, these proteins were named RadC by Haldenby [9]. However, the real roles of RadC proteins in archaea remain unclear. Based on the latest information from whole-genome sequencing, we designate the four RadA paralogs in S. tokodaii as stRadC1 (ST0579), stRadC2 (ST1830), ST0838 and ST2522.

In our previous study, we demonstrated that stRadC1 and ST0838 (corresponding to the old designations of stRad55A and stRad55B, respectively) might be involved in the recombination catalyzed by $\operatorname{RadA}$ [12]. Different from stRadC1 and ST0838, stRadC2 did not show obvious induction following DNA damage. Here, we identified the interacting proteins of stRadC 2 by pull-down assays and found that the protein can interact with both RadA and Hjc. Further investigations revealed that stRadC2 inhibited RadA-mediated recombination and facilitated the resolution of Holliday junction DNA via Hjc, the Holliday junction endonuclease. These results suggest that stRadC2 might work at the late stages of archaeal HR.

\section{Materials and methods}

\subsection{Strains, plasmids and chemicals}

The vector, pET-15b, and Escherichia coli stains, TG1 and BL21-CondonPlus (DE3)-RIL, were from Invitrogen (California, USA). S. tokodaii strain 7 was purchased from JCM (Japan Collections of Microbes, Cat. No. 10545), and was cultured at $80^{\circ} \mathrm{C}$ as described previously [15]. Taq DNA polymerase, restriction enzymes, DNA ligation kit, and T4 polynucleotide kinase were purchased from Takara (Dalian, China) and used according to the manufacturer's recommendations. $\left(\gamma-{ }^{32} \mathrm{P}\right)$ ATP was obtained from GE Healthcare (Buckinghamshire, UK). Single-stranded M13mp18 DNA was bought from Sigma (Saint Louis, MI, USA). Oligonucleotides were synthesized and gel-purified by Sangon (Shanghai, China).

For UV-irradiation of cells, cell suspensions in $10 \mathrm{mmol}$
$\mathrm{L}^{-1}$ phosphate buffer ( $\mathrm{pH}$ 7.0) were irradiated at room temperature with $200 \mathrm{~J} \mathrm{~m}^{-2}$. Then, cells were re-suspended in fresh medium and incubated at $80^{\circ} \mathrm{C}$ with shaking. After incubation, cells were harvested by centrifugation, washed twice with $10 \mathrm{mmol} \mathrm{L}^{-1}$ phosphate buffer, and stored at $-80^{\circ} \mathrm{C}$ before sample preparation.

S. tokodaii proteins, RadA (recombinase, ST0297), Hjc (Holliday junction endonuclease, ST1444), SSB (ssDNA binding protein, ST0503), pyruvate kinase (ST1617), and other DNA repair proteins used in this paper were the corresponding homologues of $S$. solfataricus and came from our laboratory stocks.

\subsection{DNA manipulations}

Plasmid and chromosomal DNAs were isolated from E. coli using the alkaline lysis method and from Sulfolobus using the lysozyme lysis method [16]. The gene encoding $S$. tokodaii stRadC2 (ST1830) was amplified by PCR using the up-stream primer 5'-GGCATATGGAGGGGTATACTGTGATT-3', and the down-stream primer 5'-TAGGATCCATAGCTACGCCCTCGATAAG-3' (Nde I and BamH I sites are underlined). The amplified fragment was digested with $N d e \mathrm{I}$ and $B a m \mathrm{H}$ I and then ligated into the expression vector $\mathrm{pET} 15 \mathrm{~b}$ to produce the recombinant vector $\mathrm{pET} 15 \mathrm{~b} /$ stRadC2.

RNA from UV- or mock-treated cells was prepared using an extraction kit from Qiagen (Hilden, Germany) according to the procedure described in the manual. The RNA was quantified spectrophotometrically and then reverse-transcribed with random primers using a First-Strand cDNA Synthesis Kit (Shennergy Biocolor Bioscience \& Technology Company, China). The subsequent PCR was carried out with the above stRadC2 primers, according to standard protocols [16].

\subsection{Biochemical assays}

\subsubsection{DNA substrates}

The 84 mer ssDNA, 84 bp double stranded (ds) DNA, and four 72 mer oligonucleotides, which could form Holliday junction DNA by annealing, were consistent with the DNA substrates used in our previous publication [17]. The DNA substrates used here were $\gamma-{ }^{32} \mathrm{P}$-labeled at their $5^{\prime}$ ends as described previously [18], and stored in TE buffer (10 $\mathrm{mmol}$ $\mathrm{L}^{-1}$ Tris- $\mathrm{HCl}, \mathrm{pH} 7.0$, and $0.5 \mathrm{mmol} \mathrm{L}{ }^{-1}$ EDTA).

\subsubsection{Pull down assays}

S. tokodaii cells were suspended in protein binding buffer (20 mmol L ${ }^{-1}$ Tris-HCl pH 7.0, 2\% glycerol, $1 \mathrm{mmol} \mathrm{L}^{-1}$ EDTA, $100 \mathrm{mmol} \mathrm{L}^{-1} \mathrm{NaCl}, 1 \mathrm{mmol} \mathrm{L}{ }^{-1}$ DTT, and 0.6 mmol $\mathrm{L}^{-1} \mathrm{PMSF}$ ) and disrupted on ice with an ultrasonicator at $600 \mathrm{~W}$ output for a total of $2 \mathrm{~min}$ and the debris was removed by centrifugation $(16000 \times g, 20 \mathrm{~min})$. The total 
proteins were treated with DNase, and then subjected to ammonium sulfate precipitation at $80 \%$ saturation to eliminate DNA fragments. The precipitate was collected by centrifugation at $25000 \times g$ for $20 \mathrm{~min}$. The resulting pellet was dissolved in binding buffer, and passed through a Ni-column. His-tagged stRadC2 was added to the flow-through fractions and incubated at $70^{\circ} \mathrm{C}$ for $20 \mathrm{~min}$. Proteins in association with stRadC2 would bind the nickel column, and were eluted with $0.8-1 \mathrm{~mol} \mathrm{~L}^{-1} \mathrm{NaCl}$ after washing the column with binding buffer. Eluted proteins were then separated on polyacrylamide gels and identified using MALDI-TOF mass spectrometry.

His-tagged stRadC2 was also used to analyze its interaction with purified recombination proteins based on a previously described protocol [16]. First, His-tagged protein was incubated with non His-tagged protein in binding buffer at $70^{\circ} \mathrm{C}$ for $20 \mathrm{~min}$, then the reaction mixture was loaded onto a Ni-NTA sepharose column (Qiagen). After washing with three column volumes of washing buffer $\left(20 \mathrm{mmol} \mathrm{L}^{-1}\right.$ Tris-HCl pH 7.0, $2 \%$ glycerol, $200 \mathrm{mmol} \mathrm{L}^{-1} \mathrm{NaCl}, 1 \mathrm{mmol}$ $\mathrm{L}^{-1}$ DTT, $40 \mathrm{mmol} \mathrm{L}^{-1}$ imidazole, and $0.6 \mathrm{mmol} \mathrm{L}{ }^{-1} \mathrm{PMSF}$ ), the proteins were eluted with $200 \mathrm{mmol} \mathrm{L}^{-1}$ imidazole and assayed by SDS-PAGE.

\subsubsection{Three-strand-exchange assay}

Reaction mixture $(20 \mu \mathrm{L})$ containing SEB reaction buffer (20 mmol L ${ }^{-1}$ Tris-HCl, $\mathrm{pH} 7.0,5$ mmol L ${ }^{-1} \mathrm{MgCl}_{2}, 2 \%$ glycerol, $1 \mathrm{mmol} \mathrm{L}^{-1}$ ATP, and ATP renew system that included $5 \mathrm{mmol} \mathrm{L}^{-1}$ phosphoenolpyruvate and $1 \mu \mathrm{g}$ pyruvate

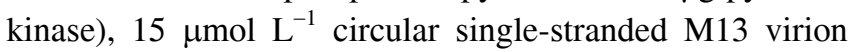
DNA, and RadA protein $\left(5 \mu \mathrm{mol} \mathrm{L}{ }^{-1}\right)$ was first incubated at $65^{\circ} \mathrm{C}$ for $5 \mathrm{~min}$. Then linear M13 dsDNA was added at a concentration of $15 \mu \mathrm{mol} \mathrm{L}{ }^{-1}$. After a second incubation at $65^{\circ} \mathrm{C}$ for $30 \mathrm{~min}$, SDS (a final concentration of $1 \%$ ) and proteinase $\mathrm{K}$ (a final concentration of $1 \mu \mathrm{g} \mu \mathrm{L}^{-1}$, Takara) were added to the reaction solution. A further incubation at $37^{\circ} \mathrm{C}$ for $40 \mathrm{~min}$ was required to fully degrade the protein in the reaction. Samples were mixed with $2 \mu \mathrm{L}$ of loading buffer composed of $50 \%$ glycerol and $0.1 \%$ bromophenol blue and resolved on a $1 \% \mathrm{TAE}$ agarose gel at $3 \mathrm{~V} \mathrm{~cm}^{-1}$.

\subsubsection{Holliday junction DNA cleavage by Hjc}

Assays were carried out in reaction buffer $\left(20 \mathrm{mmol} \mathrm{L}^{-1}\right.$ Tris- $\mathrm{HCl}$, pH 7.5, $0.5 \mathrm{mmol} \mathrm{L}{ }^{-1}$ DTT, 5 mmol L ${ }^{-1} \mathrm{MgCl}_{2}$, and $50 \mathrm{mmol} \mathrm{L}^{-1} \mathrm{NaCl}$ ) containing $10 \mathrm{nmol} \mathrm{L}{ }^{-1} \gamma-{ }^{32} \mathrm{P}$ labeled four-way DNA junction with initial equilibration at $60^{\circ} \mathrm{C}$ for $5 \mathrm{~min}$. The reactions were initiated by the addition of $5 \mathrm{nmol} \mathrm{L}{ }^{-1} \mathrm{Hjc}$ in a final reaction volume of $20 \mu \mathrm{L}$. After the indicated times, reactions were stopped by the addition of EDTA and SDS to final concentrations of $50 \mathrm{mmol} \mathrm{L}^{-1}$ and $1 \%$, respectively. Samples were then analyzed by denaturing gel electrophoresis and phosphor imaging as described [16].

\section{Results}

\subsection{Analysis of the amino acid sequence and radiation inductivity of stRadC2}

The protein stRadC2 (ST1830) is composed of 283 amino acids. Domain alignment of stRadC2 with the homologous proteins, RadA and stRadC1 from $S$. tokodaii, $\operatorname{Rad} 55$ and Rad51 from S. cerevisiae, and RadB from Thermococcus kodakarensis, is shown in Figure 1A. Compared with Ra$\mathrm{dA} / \operatorname{Rad} 51$, stRadC2 possesses the conserved ATPase core domain but lacks the N-terminal domain of RadA/Rad51, similarly to Rad55, RadB and stRadC1. In particular, the amino acid residue numbers of RadB and stRadC1 are similar to that of stRadC2. Alignment of protein sequences of RadB, stRadC1, and stRadC2 is shown in Figure 1B, and their secondary-structure elements were marked above the sequences. stRadC2 possesses low amino acid identities with stRadC1 (about 34\%) and RadB (about 18\%). Though their secondary structures are generally similar, the C-terminus of stRadC2 has two helixes, both of which are absent in RadB and one of which is missing in stRadC1. Based on the alignment, we think that stRadC2 is probably a paralog of RadA and may have particular function in $S$. tokodaii.

To investigate the role of stRadC2 in $S$. tokodaii, an RT-PCR assay of stRadC2 induction was performed after UV irradiation (Figure 1C). For 2-8 h after UV irradiation, fragmented genomic DNA was observed. Post-irradiation, RadA was induced, and pyruvate kinase (ST1617, an important enzyme in glycolysis) was apparently inhibited. Notably, the transcription of stRadC2 was inhibited between 2-6 h post-irradiation, which is different from that of stRadC1 and ST0838 [12]. However, the expression of stRadC2 was restored $8 \mathrm{hr}$ post-irradiation when RadA was induced and pyruvate kinase was inhibited.

\subsection{Pull-down analysis of stRadC2-interacting proteins from the total protein extract of $S$. tokodaii}

To analyze the function of stRadC2, the protein was expressed in E. coli strain BL21 (DE3) and isolated by heat treatment and $\mathrm{Ni}^{2+}$-NTA affinity column purification. His-tagged stRadC2 was used to isolate interacting proteins from the total protein extract of $S$. tokodaii. Seven proteins that bound to the Ni-NTA agarose beads through interaction with His-tagged stRadC2 were isolated and identified as CarB (carbamoyl phosphate synthase large subunit, ST1504), FBP (fructose-bisphosphatase, ST0318), hypothetical protein (ST1922), EF-2 (elongation factor 2, ST0437), RadA (ST0297), Hjc (Holliday junction endonuclease, ST1444), and a fragment of stRadC2 (Figure 2A). CarB is an important enzyme in the biosynthesis of arginine and the pyrimidine nucleotides, FBP is a key enzyme in gluconeogenesis, and EF-2 mediates translocation in protein synthesis 

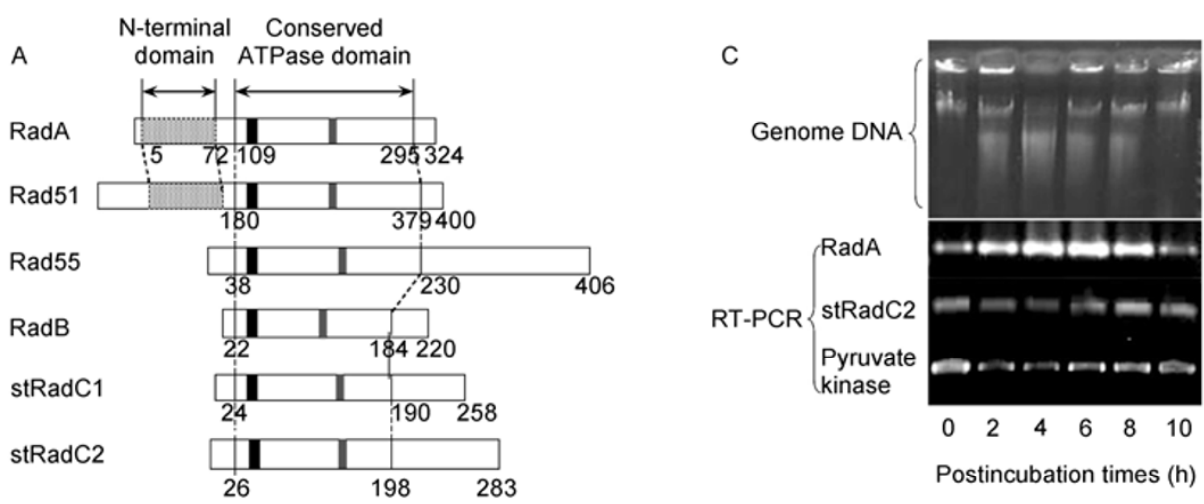

B

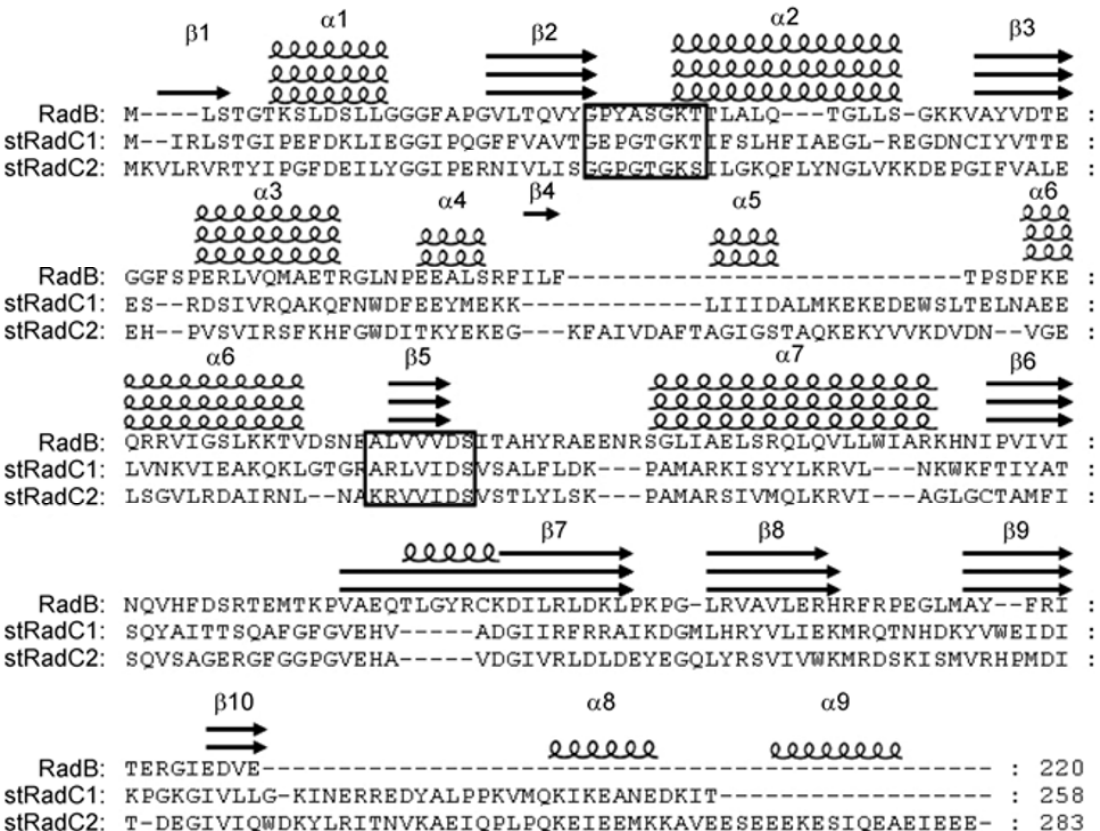

Figure 1 Domain and sequence analysis of stRadC2 from $S$. tokodaii. A, The domain structures of stRadC1, stRadC2 and RadA from $S$. tokodaii, Rad55 and Rad51 from S. cerevisiae, and RadB from T. kodakarensis. The rectangles indicate Walker A and B motifs, respectively. B, Sequence alignment of stRadC1, stRadC2 and RadB. Secondary structure elements of the three proteins are shown above the alignment. Spiral lines represent $\alpha$-helical segments and horizontal arrows represent $\beta$-sheets. C, RT-PCR assay of stRadC2 transcription after UV radiation. The irradiated cells were incubated after irradiation at $80^{\circ} \mathrm{C}$ for different times $(0,2,4,6,8,10 \mathrm{~h})$, and total RNAs were then extracted for RT-PCR assay. The transcription of RadA and pyruvate kinase was assayed as controls. The genomic DNA from irradiated cells was also isolated, digested with Hind III and EcoR I, and separated by agarose gel electrophoresis.
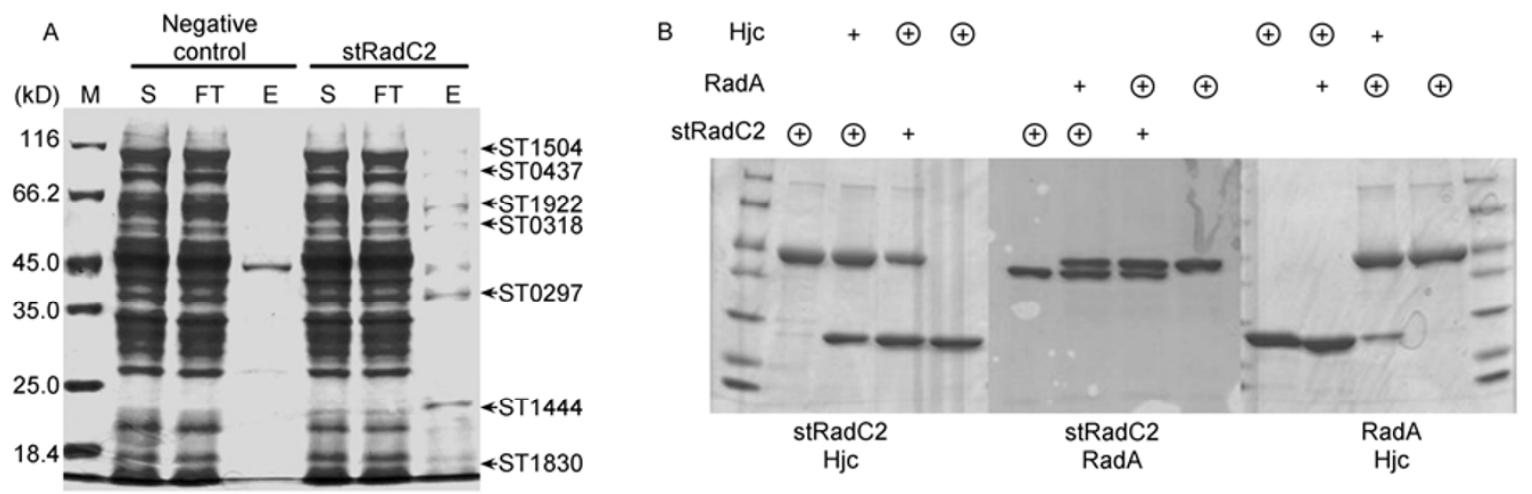

Figure 2 Interaction assays among stRadC2, RadA and Hjc proteins. A, Pull-down of stRadC2 interacting proteins from the total extract of S. tokodaii. Lane S, total protein from S. tokodaii; lane FT, proteins in flow through; lane E, proteins eluted with NaCl. Ni-NTA agarose was used as a control to exclude resin-binding proteins. B, Direct binding of purified stRadC2 protein with RadA and Hjc. His-tagged (circled+symbol) or non His-tagged (+symbol) proteins were used to investigate reciprocal interactions. 
and facilitates the events of translational elongation. All three of these proteins are needed for normal cellular metabolism [19]. RadA and Hjc are DNA damage repair proteins, which are involved in DNA recombination and Holliday-junction cleavage, respectively. The result that stRadC2 could pull down these proteins suggests that stRadC2 might be involved in both metabolism and DNA repair. These proteins may also bind the column non-specifically. Surprisingly, only a fragment but not the full length stRadC2 was found in the pull-down sample, a phenomenon that needs further investigation. Here, our focus was primarily on the known repair proteins, $\operatorname{RadA}$ and $\mathrm{Hjc}$, to study the role of stRadC2 in archaeal DNA repair.

The interaction among purified stRadC2, RadA, and Hjc was examined in vitro. As shown in Figure 2B, stRadC2 could bind with RadA and Hjc, and only a minor interaction was found between RadA and Hjc. This result suggests that the three proteins may interact and form a complex, although other methods such as gel filtration and yeast two hybrid are needed to verify the protein interactions and formation of the complex.

\subsection{StRadC2 is a negative regulator of $\operatorname{RadA}$ recombi- nation}

To further understand the interaction between stRadC2 and RadA (or Hjc), biochemical assays of stRadC2 on the activities of RadA and Hjc were carried out. As shown in Figure $3 \mathrm{~A}$, RadA protein could enhance the homologous strand exchange reaction (lane 2), while stRadC2 protein could not (lane 1). With the addition of a gradient of stRadC2 protein (from 1 to $7 \mu \mathrm{mol} \mathrm{L} \mathrm{L}^{-1}$ ) to the strand exchange reaction, the amount of reaction product clearly decreased (lanes 3-6).
Thus, purified stRadC2 could inhibit the recombination activity of RadA.

To understand the inhibition mechanism, the effect of the order in which proteins were added on the DNA strand exchange activity was analyzed. When RadA was incubated with DNA before $\mathrm{stRadC2}$, the level of recombination product was not significantly reduced (lane 3 in Figure 3B). However, when stRadC2 was incubated with DNA before $\mathrm{RadA}$, the level of product was clearly reduced (lane 4 in Figure 3B), suggesting that stRadC2 blocked the binding of RadA to ssDNA. When the two proteins were mixed with each other before incubation with DNA, the recombination was inhibited dramatically (lane 5 in Figure 3B). Thus, the interaction of stRadC2 and $\operatorname{RadA}$ repressed their binding to ssDNA.

To further confirm that the stRadC2 inhibition of RadA recombination is related to their binding to DNA, gel shift assays with $\gamma-{ }^{32} \mathrm{P}$ labelled ssDNA were performed to test the interactions between RadA and stRadC2 (Figure 3C). When RadA was pre-incubated with ssDNA before the addition of stRadC2, little free ssDNA was released (lane 3). When tRadC2 was added first or the two proteins were mixed before adding to ssDNA, a greater amount of free ssDNA was released (lanes 4 and 5). Thus, the stRadC2 inhibition of RadA recombination activity is likely to be mainly caused by interference of the binding of RadA to ssDNA.

\section{4 stRadC2 could promote the Holliday junction cleavage activity of $\mathrm{Hjc}$}

S. tokodaii Hjc is a homologue of S. solfataricus and P. furiosus Hjc proteins that have Holliday junction DNA cleavage activity $[18,20]$. Here, a standard in vitro cleavage
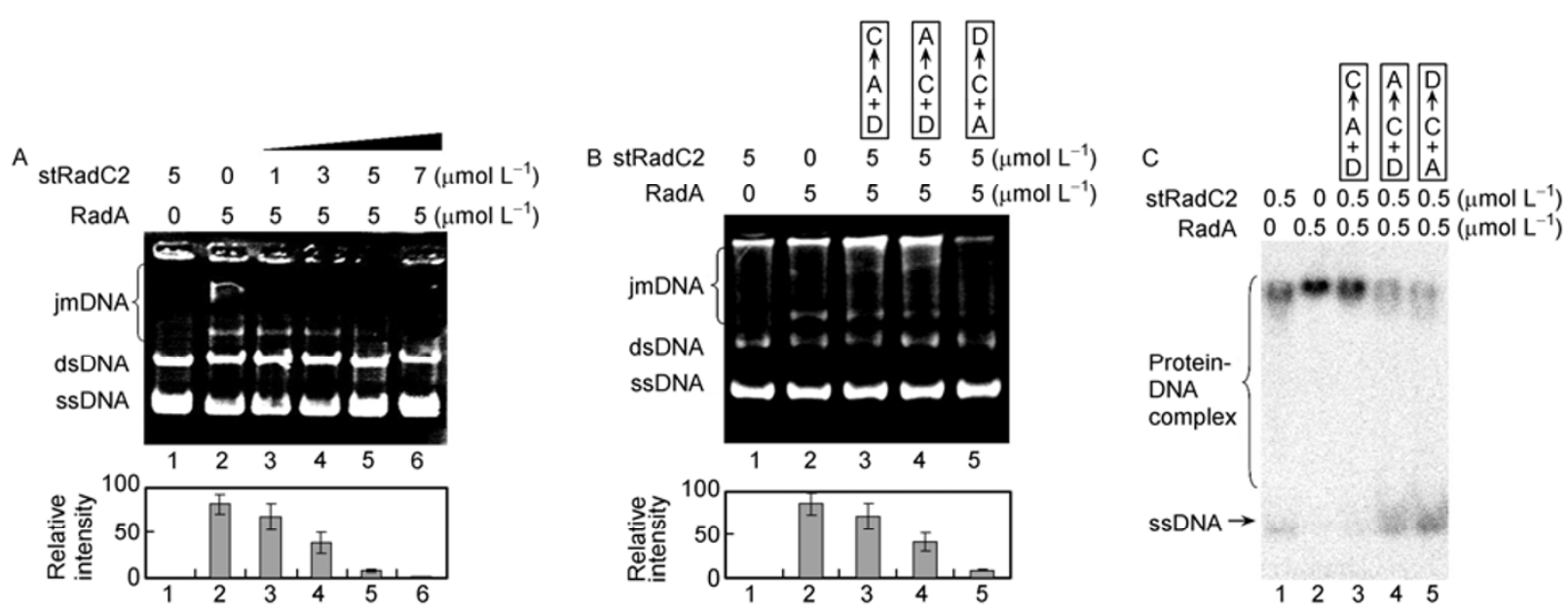

Figure 3 The effect of stRadC2 on the DNA strand exchange activity of RadA. The reaction was carried out as described in Materials and methods, and the product (molecular junction DNA), single strand DNA, and double strand DNA are denoted as jmDNA, ssDNA and dsDNA, respectively. A, A concentration gradient of stRadC2 inhibited the DNA strand exchange catalyzed by RadA. Lane 1, stRadC2; lane 2, RadA; lanes 3-6, RadA and the stRadC2 gradient (from 1 to $7 \mu \mathrm{mol} \mathrm{L}{ }^{-1}$ ). B. The effect of the order in which stRadC2 was added to the strand exchange reaction. C, Gel shift assay of the binding of the two proteins to $\gamma-{ }^{32} \mathrm{P}$ labeled ssDNA. Lane 1, stRadC2 protein alone; lane 2, RadA protein alone; 1 lane 3, RadA pre-incubated with ssDNA; lane 4, stRadC2 pre-incubated with ssDNA; lane 5, the two proteins were pre-incubated before adding DNA. The adding order of stRadC2 (C), RadA (A) and ssDNA (D) is also marked above the figure. The relative intensities of the product bands were measured using a Gel Imager System, and the values are the averages $( \pm$ SD) from three independent experiments. 
assay was used with a synthetic four-way junction as a substrate, and the cleavage efficiency of $\mathrm{Hjc}$ was examined in the presence of a concentration gradient of stRadC2 (Figure 4A). The results showed that stRadC2 promoted the junction DNA cleavage activity of Hjc. As the concentration of stRadC2 increased, so did the cleavage rate. At $8 \mathrm{nmol} \mathrm{L}^{-1}$ stRadC2, about $50 \%$ of Holliday junction DNA was resolved.

Because stRadC2 could bind to DNA, it is necessary to examine whether stRadC2 facilitated Hjc activity by way of its DNA binding ability. The effect of the order in which proteins were added on the cleavage reaction was analyzed with $5 \mathrm{nmol} \mathrm{L}{ }^{-1} \mathrm{Hjc}$ and $8 \mathrm{nmol} \mathrm{L}^{-1}$ stRadC2. The results showed that the cleavage rates did not show any significant changes with respect to the order in which proteins were added (Figure 4B). The effect of stRadC2 on $\mathrm{Hjc}$ binding to Holliday junction DNA labelled with $\gamma-{ }^{32} \mathrm{P}$ was then investigated, and the result showed that the release of junction DNA increased as the concentration of stRadC2 increased (Figure 4C). The activation of stRadC2 on $\mathrm{Hjc}$ did not result from its facilitation of binding $\mathrm{Hjc}$ to DNA, and the real mechanism needs further study.

\section{Discussion}

This study is part of an effort to understand the functions of RadA and its paralogs in the hyperthermophilic archaeon, $S$. tokodaii. Similar to RecA in bacteria, Rad51 in eukaryotes, and RadA in halophilic or methanogenic archaea, Sulfolobus RadA is also a DNA damage-inducible recombination repair enzyme [21]. To face the challenges from their hyperthermic environment, Sulfolobus express RadA at high basal levels, which is beneficial to the preservation of their genetic integrity. However, like a two-edged sword, excessive recombinase will likely result in inappropriate chromosomal crossing over and endanger genomic stability [22]. In bacteria, RecX and DinI were found as anti-recombinases to limit excessive recombination activity by binding to RecA protein directly $[23,24]$. In eukaryotic cells, several proteins, including Rad51 paralogs that interact with the human Rad51 protein, inhibit strand exchange and replication fork
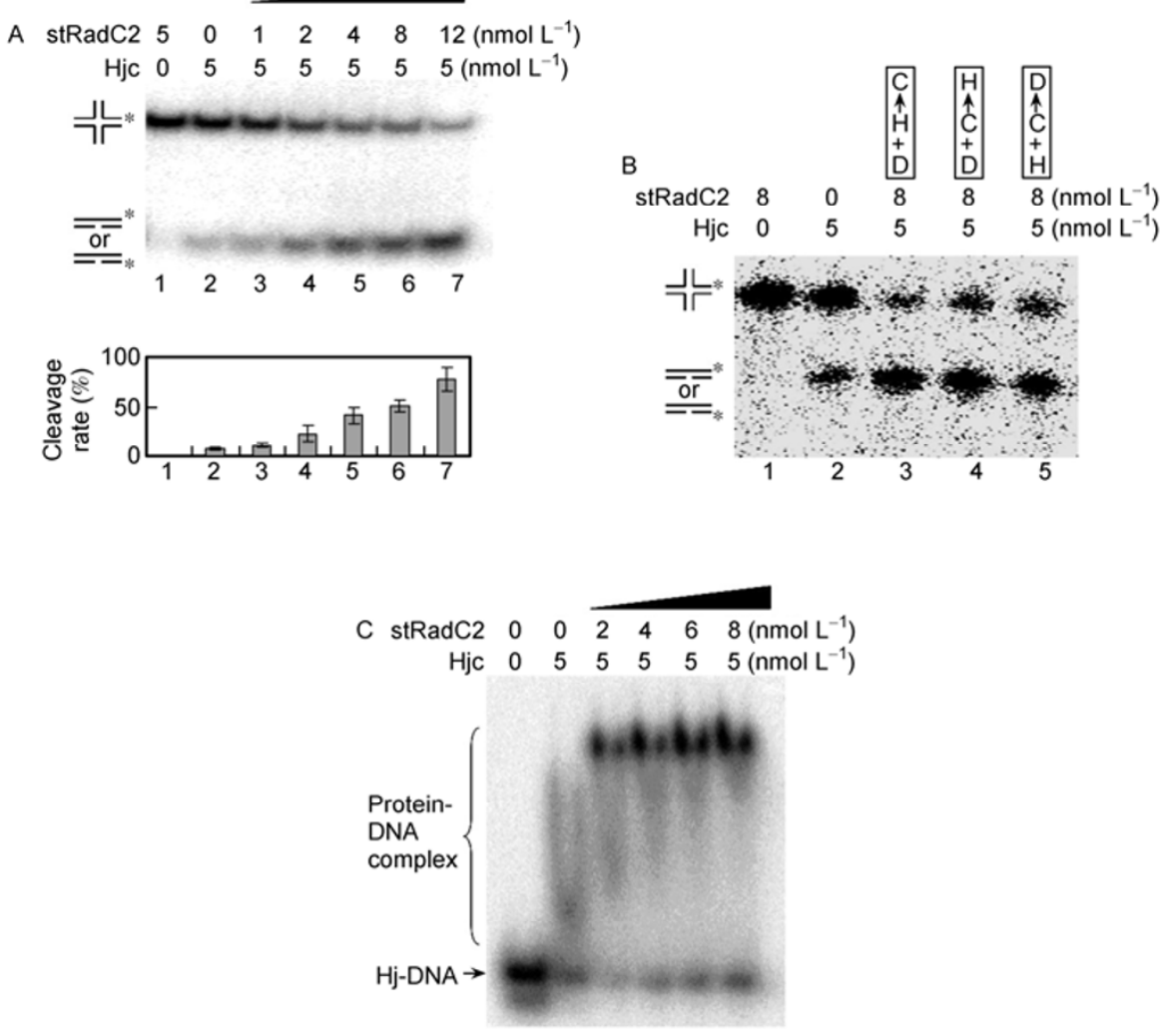

Figure 4 Effect of stRadC2 on junction DNA cleavage and binding activity of Hjc. Holliday junction DNA cleavage was carried out in reaction buffer (20 mmol L ${ }^{-1}$ Tris- $\mathrm{HCl}$, pH 7.5, $0.5 \mathrm{mmol} \mathrm{L}{ }^{-1} \mathrm{DTT}, 5 \mathrm{mmol} \mathrm{L} \mathrm{MgCl}_{2}$, and $50 \mathrm{mmol} \mathrm{L}^{-1} \mathrm{NaCl}$ ) with $10 \mathrm{nmol} \mathrm{L}^{-1} \gamma^{32} \mathrm{P}$ labeled four-way junction DNA. The binding assay was carried out in the Holliday junction DNA cleavage buffer without $\mathrm{MgCl}_{2}$. A, A gradient of stRadC2 facilitates the cleavage activity of Hjc. $\mathrm{B}$, The effect of the order in which protein was added on junction DNA cleavage by Hjc. Lane 1, stRadC2 alone; lane 2, Hjc alone; lane 3, Hjc pre-incubated with DNA; lane 4, stRadC2 pre-incubated with DNA; lane 5, the two proteins added at the same time. C, Gel shift assay of the effect of a concentration gradient of stRadC2 on Hjc protein binding to junction DNA. The bands were scanned and the cleavage rate (product DNA/total DNA) was calculated. Values are the average $( \pm \mathrm{SD})$ from six independent experiments. 
regression promoted by human Rad51 [25]. Euryarchaeal RadB and crenarchaeal stRadC2 are known to inhibit RadA recombination activity, which has enriched our knowledge on crenarchaeal DNA recombination. In addition, stRadC2 interacts with $\mathrm{Hjc}$ and facilitates the resolution of Holliday DNA junctions, which is more similar to its eukaryotic equivalents [3-6] than to its euryarchaeal ones [9,10].

At the late stages of DNA recombination, the cell ends DNA repair and starts normal metabolism. Checkpoint proteins are involved in the switch between DNA damage response and normal metabolism [26]. In Figure 2A, stRadC2 could pull down both DNA repair proteins and normal metabolism-related proteins, suggesting that stRadC2 might be involved in both metabolism and DNA repair. stRadC2 is not induced by DNA damage, negatively regulates DNA recombination, and is up-regulated $8 \mathrm{hr}$ post-irradiation, which indicates that stRadC2 might function at the late stages of DNA repair.

This work was supported by the National Natural Science Foundation of China (Grant Nos. 3093002 and 30870046 to Shen YuLong, and 30700011 to Sheng DuoHong) and the Promotive Research Fund for Excellent Young and Middle-aged Scientists of Shandong Province (Grant No. BS2010SW014 to Sheng DuoHong).

1 McCready S J, Osman1 F, Yasui A. Repair of UV damage in the fission yeast Schizosaccharomyces pombe. Mutat Res, 2000, 451: 197-210

2 Sung P. Yeast Rad55 and Rad57 proteins form a heterodimer that functions with replication protein A to promote DNA strand exchange by Rad51 recombinase. Genes Dev, 1997, 11: 1111-1121

3 Mozlin A M, Fung C W, Symington L S. Role of the Saccharomyces cerevisiae Rad51 paralogs in sister chromatid recombination. Genetics, 2008, 178: 113-126

4 Kawabata M, Kawabata T, Nishibori M. Role of recA/RAD51 family proteins in mammals. Acta Med Okayama, 2005, 59: 1-9

5 Thacker J. A surfeit of RAD51-like genes? Trends Genet, 1999, 15: 166-168

6 Miller K A, Sawicka D, Barsky D. Domain mapping of the Rad51 paralog protein complexes. Nucleic Acids Res, 2004, 32: 169-178

7 Takata M, Sasaki M S, Tachiiri S, et al. Chromosome instability and defective recombinational repair in knockout mutants of the five Rad51 paralogs. Mol Cell Biol, 2001, 21: 2858-2866

8 Kelman Z, White M F. Archaeal DNA replication and repair. Curr Opin Microbiol, 2005, 8: 669-676

9 Haldenby S, White M F, Allers T. RecA family proteins in archaea:
RadA and its cousins. Biochem Soc Trans, 2009, 37: 102-107

10 Komori K, Miyata T, DiRuggiero J, et al. Both RadA and RadB are involved in homologous recombination in Pyrococcus furiosus. $\mathrm{J}$ Biol Chem, 2000, 275: 33782-33790

11 Guy C P, Haldenby S, Brindley A, et al. Interactions of RadB, a DNA repair protein in archaea, with DNA and ATP. J Mol Biol, 2006, 358: 46-56

12 Sheng D, Zhu S, Wei T, et al. The in vitro activity of a Rad55 homologue from Sulfolobus tokodaii, a candidate mediator in RadA-catalyzed homologous recombination. Extremophiles, 2008, 12: 147-157

13 McRobbie A M, Carter L G, Kerou M, et al. Structural and functional characterisation of a conserved archaeal RadA paralog with antirecombinase activity. J Mol Biol, 2009, 389: 661-673

14 Abella M, Rodríguez S, Paytubi S, et al. The Sulfolobus solfataricus radA paralogue sso0777 is DNA damage inducible and positively regulated by the Sta1 protein. Nucleic Acids Res, 2007, 35: 6788-6797

15 Brock T D, Brock K M, Belly R T, et al. Sulfolobus: A new genus of sulfur-oxidizing bacteria living at low $\mathrm{pH}$ and high temperature. Arch Mikrobiol, 1972, 84: 54-68

16 Ausubel F M, Brent R, Kingston R E. Short Protocols in Molecular Biology. 3rd ed. New York: Cold Spring Harbor Laboratory Press, 1995

17 Zhang S, Wei T, Hou G, et al. Archaeal DNA helicase HerA interacts with Mre11 homologue and unwinds blunt-ended double-stranded DNA and recombination intermediates. DNA Repair (Amst), 2008, 7: 380-391

18 Komori K, Sakae S, Fujikane R, et al. Biochemical characterization of the Hjc Holliday junction resolvase of Pyrococcus furiosus. Nucleic Acids Res, 2000, 28: 4544-4551

19 Kawarabayasi Y, Hino Y, Horikawa H, et al. Complete genome sequence of an aerobic thermoacidophilic crenarchaeon, Sulfolobus tokodaii strain7. DNA Res, 2001, 8: 123-140

20 Kvaratskhelia M, White M F. Two Holliday junction resolving enzymes in Sulfolobus solfataricus. J Mol Biol, 2000, 297: 923-932

21 Sheng D, Zhu S, Li M, et al. Cloning, expression and radiation inducibility of RadA from the hyperthermophilic archaeon Sulfolobus tokodaii. Wei Sheng Wu Xue Bao, 2008, 48: 317-22

22 Klein H L. The consequences of Rad51 overexpression for normal and tumor cells. DNA Repair (Amst), 2008, 7: 686-693

23 Venkatesh R, Ganesh N, Guhan N, et al. RecX protein abrogates ATP hydrolysis and strand exchange promoted by RecA: insights into negative regulation of homologous recombination. Proc Natl Acad Sci USA, 2002, 99: 12091-12096

24 Lusetti S L, Drees J C, Stohl E A, et al. The DinI and RecX proteins are competing modulators of RecA function. J Biol Chem, 2004, 279: 55073-55079

25 Yoon D, Wang Y, Stapleford K, et al. P53 inhibits strand exchange and replication fork regression promoted by human Rad51. J Mol Biol, 2004, 336: 639-654

26 Houtgraaf J H, Versmissen J, van der Giessen W J. A concise review of DNA damage checkpoints and repair in mammalian cells. Cardiovasc Revasc Med, 2006, 7: 165-172

Open Access This article is distributed under the terms of the Creative Commons Attribution License which permits any use, distribution, and reproduction in any medium, provided the original author(s) and source are credited. 